\title{
Effect of Powder Recycling in Laser-based Powder Bed Fusion of Ti-6Al-4V
}

\author{
Lucia Denti $^{1}$, Antonella Sola ${ }^{1}$, Silvio Defanti ${ }^{1}$, Corrado Sciancalepore $^{2}$, Federica Bondioli ${ }^{3}$ \\ ${ }^{1}$ Department of Engineering "Enzo Ferrari” (DIEF), University of Modena and Reggio Emilia, via P. Vivarelli 10, 41125 \\ Modena. Italy. E-mail: lucia.denti@unimore.it, antonella.sola@unimore.it, silvio.defanti@unimore.it \\ ${ }^{2}$ INSTM, Research Unit of Modena, Department of Engineering Enzo Ferrari (DIEF), University of Modena and Reggio \\ Emilia, via P. Vivarelli 10, 41125 Modena. Italy. E-mail: corrado.sciancalepore@unimore.it \\ ${ }^{3}$ Department of Applied Science and Technology (DISAT), Polytechnic University of Turin, Corso Duca degli Abruzzi \\ 24, 10129 Torino, Italy. E-mail: Federica.bondioli@polito.it
}

\begin{abstract}
Additive manufacturing (AM) has shown promise to process parts for end-use applications, however stringent requirements must be fulfilled in terms of reliability and predictability. The expensiveness of raw materials for AM, especially for metal-based Powder Bed Fusion (PBF), brings about the need for a careful recycling of powder, but the effect of powder reuse on both processing conditions and final part performance is still the focus of intensive research in the open literature. Although ASTM F2924-14 specifies the virgin-to-used powder ratio to be introduced to manufacture titanium-6aluminum-4vanadium (Ti-6Al-4V) components by PBF, a deeper understanding of the effect of powder recycling on the mechanical properties of finished parts is expected to foster a more efficient and safe reuse. The present contribution is therefore addressed to investigate the consequence of Ti6Al-4 V powder recycling on the flowability, particle size distribution and morphology of the feedstock material as well as on the density and tensile performance of built parts. In order to quantify the recyclability of powders, a new "average usage time" (AUT) parameter is defined to account for both the real usage time of the powder and the virgin-to-used powder mixing ratio. The new parameter, whose applicability can be readily extended to any kind of feedstock powder, offers a significant contribution to achieve a more consistent and economical recycling of raw materials for PBF processing.
\end{abstract}

Keywords: Laser-based powder bed fusion; Powder; Recycling; Ti6Al4V; Mechanical properties.

\section{Introduction}

At present, powder bed fusion (PBF) is the most important additive manufacturing (AM) technique to produce metal parts [1]. Among the available metal powders that can be used for PBF, Ti-6Al-4V plays a fundamental role to build almost ready-to-use parts. In fact, this titanium alloy benefits from its high mechanical propertiesto-specific mass ratio that makes it suitable for motorsport, aerospace and medical applications [2-4].

The increasing success of PBF in industry is fostered by a continuous scientific research that is addressed to optimize the process parameters. A fine tuning of the processing conditions is very important to maximize productivity and, at the same time, to ensure the consistency of the built parts and to improve their performance.

However, metallic powders for PBF can be very expensive, since they may cost up to $700 \mathrm{USD} / \mathrm{kg}$. For example, the cost of Nickel Alloy 718 powder for AM has been recently estimated to be about $90 \mathrm{USD} / \mathrm{kg}$. Moreover, only 3 to $5 \%$ of the powder that exits the dispenser is actually used to build the part. Without recycling, the real cost of the powder in the finished part rises therefore to $3000 \mathrm{USD} / \mathrm{kg}$ [5]. Powder recycling becomes even more imperative when Ti-6Al-4V powder is involved, since Ti-6Al-4V feedstock for AM may cost up to 400 $\mathrm{USD} / \mathrm{kg}$. As a consequence, if loose particles are not reused, the cost of built parts is expected to be prohibitive, thus obliterating the economical advantage of near-netshape manufacturing. Powder recycling is essential in order to limit the final cost of PBF processing. Nonetheless, recycling is not straightforward, since recovering operations and repeated consolidation processes during manufacturing are likely to engender chemical and morphological changes in the reused particles and to alter the properties of the final workpieces [6]. According to ASTM F2924-14 [7], the feedstock powder is indeed the most important parameter that must be controlled during manufacturing in order to govern the performance of final built parts. It is known in fact that the powder characteristics, in terms of particle size and particles size distribution, morphology, chemical and mineralogical composition, deeply influence the correct development of PBF processing and the consequent properties of finished parts [8].

Some contributions have already been published in the literature concerning the reuse of feedstock powders in PBF techniques. For example, Seyda et al. [9] investigated the effect of possible aging phenomena in laserbased powder bed fusion (L-PBF) of Ti-6Al-4V. However, only twelve recycling steps were considered. Moreover, the duration of each job was not specified and the powder was exposed to atmospheric environment during sieving operations. This approach was useful to define repeatable processing conditions. Nonetheless, if the real industrial practice is considered, hundreds of recycling steps are often performed, building processes may have variable duration and sieving operations are conducted under protective gas environment [9]. Tang et al. discussed the effect of Ti-6Al-4V powder reuse in selective electron beam melting. To this aim, Tang et al. processed repeatedly the same powder, without any addition of virgin supply. As a consequence, the powder was enough to 
complete just 21 jobs, whose time duration was not specified [10].

With respect to the available literature, the present contribution is addressed to analyze the consequence of Ti-6Al-4V powder recycling on the mechanical properties of L-PBF parts produced in an industrial-like setting. In order to reproduce realistic industrial operations, more than 100 recycling steps were completed, virgin powder was added if required to complete the new job and all manipulation operations were performed in protective atmosphere. As a means to reveal the combined effect of repeated powder reuse and virgin powder addition, a new "average usage time" (AUT) parameter was introduced that takes into account the real duration of powder handling, rather than the number of recycling steps. Though defined here for L-BPF, the new parameter can be readily applied also to other processing methods thus offering a significant contribution to the improvement of AM efficiency and economical impact.

\section{Materials and methods}

\subsection{Virgin powder}

Commercial Ti-6Al-4V powder (EOS Titanium Ti64, EOS GmbH, Germany) was used to perform the entire experimental activity.

The powder morphology and cross-sectional microstructure were observed in the field-free mode (secondary electron imaging) with a field emission gun scanning electron microscope (FEGSEM, Nova NanoSEM 450, FEI Quanta, The Netherlands) equipped with an Xray energy dispersion spectroscopy system (X-EDS Bruker QUANTAX-200, Germany) for chemical micro-analysis. As for the cross sectional investigation, powder particles were embedded in resin, then the Ti-6Al-4V powder/resin pieces were cut and polished up to $0.25-\mu \mathrm{m}$ diamond paste on a porous neoprene cloth. The polished surface of the Ti-6Al-4V powder/resin pieces was treated with the Keller's reagent for about 10 seconds, in order to show the internal microstructure of the powder grains.

X-ray diffraction ("XRD”, X'pert PRO, PANalytical, Almelo, Netherlands) was carried out in the $30-85^{\circ} 2 \theta$ angular range by using the $\mathrm{CuK} \alpha$ radiation $(\lambda \mathrm{K} \alpha 1=1.5406$ $\AA, \lambda \mathrm{Ka} 2=1.5444 \AA$ ); the scans were acquired with a step size of $0.0167^{\circ} 2 \theta$ and counting time of $500 \mathrm{~s}$. The diffractograms were analyzed and fitted by means of the Rietveld method in order to establish a first quantitative estimation of the phase content.

The particle size distribution was analyzed with a laser granulometer (Mastersizer 2000 Ver. 5.22, Malvern Instruments Ltd., Malvern, UK) according to ASTM B822-10 [11]. The particle size analysis was conducted in wet mode, using water as dispersing medium.

The flow rate of the powder was measured with the Hall and Carney calibrated funnels according to ASTM B213-17 [12] and to ASTM B964-16 [13], respectively. In order to gain a deeper insight into the flow properties of the powder, the flow function was obtained according to ASTM D7891-15 [14]. For collecting the shear data to describe the powder flowability, shear cell tests were performed at different consolidation stresses $(3,6,9,15 \mathrm{MPa}$ respectively) by using the universal powder rheometer FT4 (Freeman Technology, Malvern, UK).

Whenever possible, the tests were repeated on the virgin powder and on the recycled powder at the end of the recycling test, which corresponded to an AUT of 1750 hours as defined in the following section 2.2.

\subsection{Powder recycling strategy and definition of the average usage time, $\boldsymbol{A U T}$}

At the beginning of the experiment, the powder tank was filled to its maximum capacity $q_{0}$. At the end of the first job, all the unfused particles, including the loose powder remaining in the build chamber and the overflow, were removed from the L-PBF machine, thoroughly mixed and sieved through a $80 \mu \mathrm{m}$ sieve under inert gas conditions. Then, the powder was weighted and its mass $q_{1}$ was compared to the mass required for the following job. If the reused powder was enough, the new job was started. The same procedure was applied to all subsequent jobs until the mass of the residual powder became insufficient to complete the following job. At that point, the tank was filled again using the available reused powder and adding some virgin powder to restore the maximum capacity $q_{0}$ of the powder tank. Due to the repeated recycling, the usage time of the powder increased progressively, until new virgin powder was added.

In order to account for the effect of both repeated recycling and refilling, the average usage time, $A U T$, is defined as follows.

- $\quad$ For virgin powder, $A U T$ is assumed to be 0 .

- At the beginning of the experimental activity, the tank is filled with virgin powder and therefore $A U T(0)=0$. It is assumed that the initial amount of powder loaded in the tank, that corresponds to the maximum tank capacity $q_{0}$, is enough to complete the first job.

- $\quad$ At the end of the $i$-th job, whose duration is $t_{i}$ hours, if the residual powder $q_{i}$ in the tank is sufficient to complete the following $(i+1)$-th job, no virgin powder is added and the value for $A U T$ at the end of the $i$-th job, namely $\operatorname{AUT}(i)$, is calculated by adding $t_{i}$ to the previous value of $A U T$, namely $A U T(i-1)$, which had been determined at the end of the preceding (i-1)-th job. In other words, AUT(i) is calculated according to the expression

$$
\operatorname{AUT}(i)=\operatorname{AUT}(i-1)+t_{i}
$$

- Otherwise, if the residual powder $q_{i}$ at the end of the $i$-th job is not sufficient to complete the following $(i+1)$-th job, a mass $\left(q_{0}-q_{i}\right)$ of virgin powder is added to refill the tank and the value for $A U T$ is calculated according to a modified rule of mixture:

$$
\operatorname{AUT}(i)=\frac{q_{i} \cdot\left[\operatorname{AUT}(i-1)+t_{i}\right]+\left(q_{0}-q_{i}\right) \cdot A U T_{\text {add }}}{q_{0}}
$$

where $A U T_{\text {add }}$ is the $A U T$ of the added powder. Since, as previously stated, $A U T$ is assumed to 
be 0 for virgin powder (and hence $A U T_{a d d}=0$ ), the previous equation (1.b) can be simplified to the expression:

$$
\operatorname{AUT}(i)=\frac{q_{i} \cdot\left[\operatorname{AUT}(i-1)+t_{i}\right]}{q_{0}}
$$

As $q_{i}<q_{0}, A U T(i)$ calculated according to equation (1.b') is lower than $A U T(i)$ calculated according to equation (1.a), as expected due to the addition of new (virgin) powder to the reused one.

In the present experiment, the recycling process was repeated up to 100 times, corresponding to an AUT of 1750 hours, to simulate real industrial practice.

\subsection{PBF parameters}

An EOS M290 machine was operated by applying standard process parameters for EOS Ti-6Al-4V powder (EOS Parameter set: Ti64_Performance_M291 1.10 [15]), as reported in Tab. 1.

Tab. 1 Recommended parameters for $L-P B F$ processing of Ti-6Al-4V powders

\begin{tabular}{lc}
\hline Parameter & Value \\
\hline Laser power & $280[\mathrm{~W}]$ \\
Scan speed & $1200[\mathrm{~mm} / \mathrm{s}]$ \\
Layer thickness & $30[\mu \mathrm{m}]$ \\
Hatch distance & $140[\mu \mathrm{m}]$ \\
\hline
\end{tabular}

Specific positions within the build platform were numbered from 1 to 9 as shown in Fig. 1a.

(a)

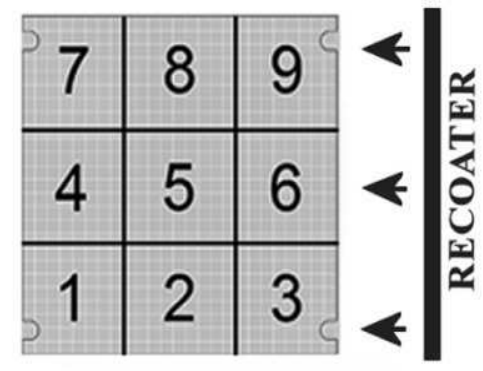

(b)

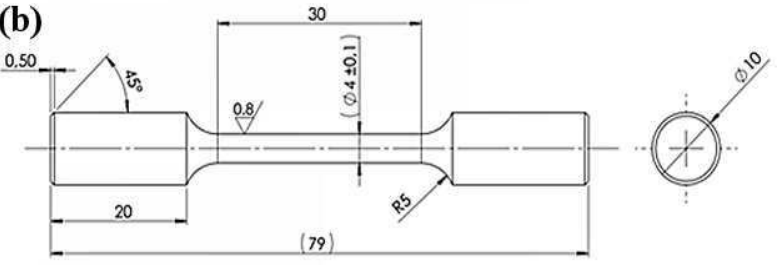

Fig. 1 Relative positions of the built parts within the build platform (a) and geometry of the tensile specimens as detailed in UNI-EN 6892-1 [16](b)

Each job included the production of complicated built parts, coherently with ordinary industrial practice, as well as the production of cubic specimens and tensile ones for subsequent characterization. Cubes of $10 \mathrm{~mm}$ by side were produced for the evaluation of density and for SEM inspection of the cross-sectional microstructure. Cylindrical specimens were manufactured for tensile tests according to the geometry specified in UNI-EN ISO 6892-1 [16], as illustrated in Fig. 1b. The orientation of the tensile specimens in the build chamber was always parallel to the build platform.

For all built parts, stress relief heat treatment was performed at $710^{\circ} \mathrm{C}$ for 2 hours, then the specimens were cooled down slowly to $500^{\circ} \mathrm{C}$ and removed from the furnace. Tensile specimens were machined in order to exclude the potential effects of uneven surface finish.

\subsection{Built parts characterization}

The density of the cubic specimens was measured by means of the Archimedes' method. The same cubes were cross-cut and polished to evaluate their microstructure and chemical composition by SEM and X-EDS, respectively.

The XRD analysis and Rietveld fitting were repeated on the cubic samples in order to verify any possible changes in phase composition that may be induced by L-PBF processing and by stress relief heat treatment.

Tensile tests were performed with an Instron 5567 testing machine equipped with a $30 \mathrm{kN}$ load cell and an Instron 2630 extensometer with a gauge length of $25 \mathrm{~mm}$. The accuracy for force measurement was $\pm 30 \mathrm{~N}$, which corresponded to a stress accuracy of $\pm 2.4 \mathrm{MPa}$ for $4 \mathrm{~mm}$ diameter specimens, while the accuracy for elongation was $0.2 \%$. The test speed was set to $2 \mathrm{~mm} / \mathrm{min}$.

The results were processed through statistical analysis using Statsoft Statistica 8.0 to calculate the correlation coefficients between the $A U T$ of the powder and the mechanical properties of the built parts.

\section{Results and discussion}

\subsection{Characterization of the virgin feedstock powder}

As shown in Fig. 2, the SEM observation confirmed that the morphology of the virgin powder was nearly spherical, apart from few slightly elongated particles. A very small amount of broken particles could be detected (Fig. 2a and b). The prevailing presence of nearly spherical particles is coherent with the need for good flowability that is typical of PBF techniques [17]. The cross section of the feedstock particles was fully-dense, without any visible internal cavities or holes (Fig. 2c). A feather-like fine microstructure was associated to the likely co-existence of two different phases (Fig. 2d).
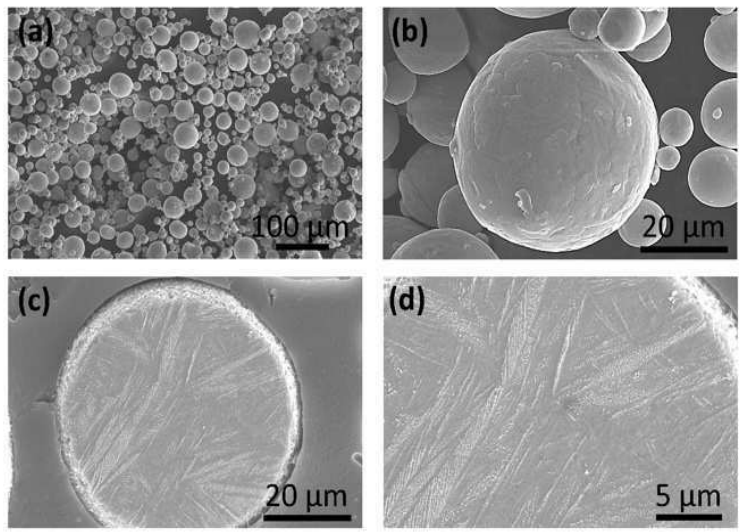

Fig. 2 Morphology $(a, b)$ and polished cross section (c, d) of the virgin feedstock powder. 
The local chemical microanalysis (Fig. 3a) revealed the presence of $\mathrm{Ti}, \mathrm{Al}$, and $\mathrm{V}$. This result was predictable for Ti-6Al-4V powders that were handled under a protective atmosphere.

The structure of the powder sample analyzed via XRD

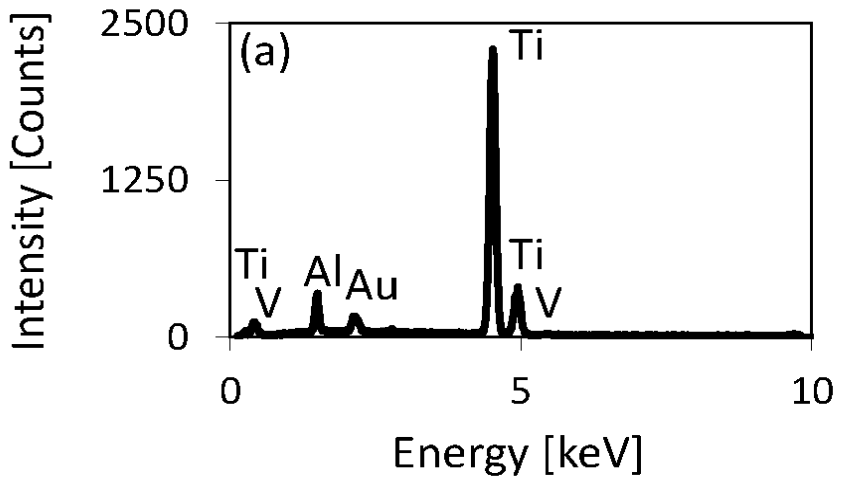

was compatible with the presence of two crystallographic titanium phases, namely an $\alpha$ phase and an $\alpha$ ' distorted phase, as evidenced by fitting the experimental diffraction pattern by means of the Rietveld method (Fig. 4a).

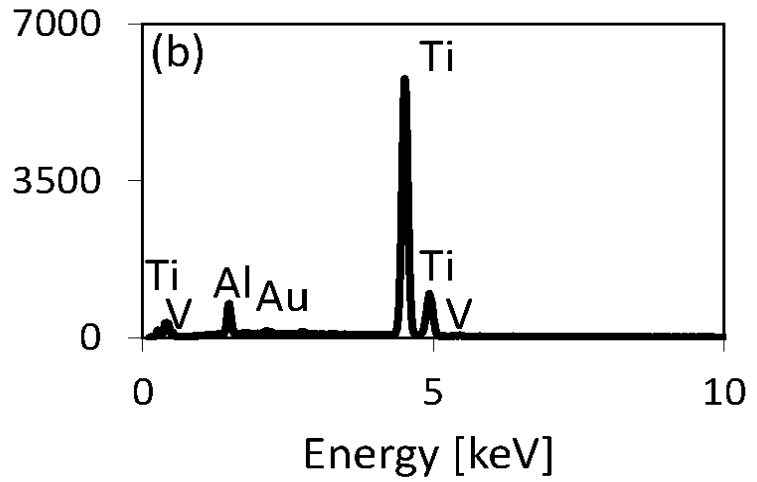

Fig. $3 X$-EDS spectra obtained on the cross section of the powder feedstock (a) and of the printed sample (b).
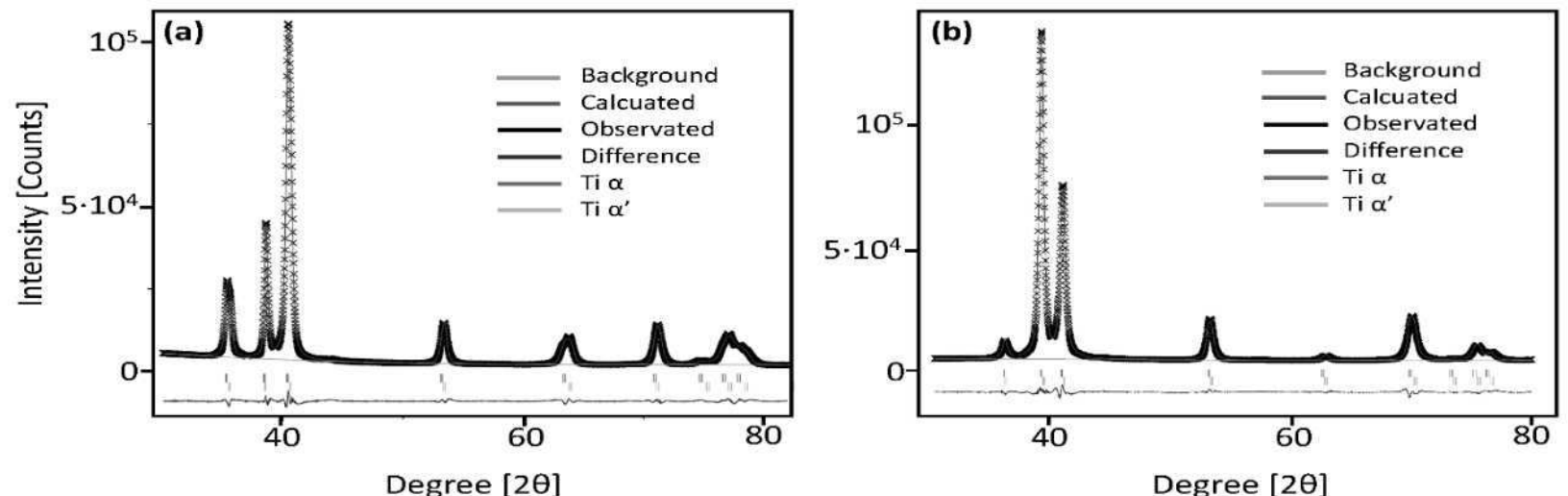

Fig. 4 Diffraction patterns of the powder feedstock (a) and of the printed sample (b) fitted by the Rietveld method.

Tab. 2 Particle size distribution and flowability of the virgin powder and the same powder after an AUT of $1750 \mathrm{~h}$

\begin{tabular}{llcccc}
\hline Powder batch & \multicolumn{2}{c}{ Particle size distribution $[\boldsymbol{\mu m}]$} & \multicolumn{2}{c}{ Flowability [g/s] } \\
& $\boldsymbol{d 1 0}$ & $\boldsymbol{d 5 0}$ & $\boldsymbol{d 9 0}$ & Hall & Carney \\
\hline Virgin powder & 24.6 & 38.6 & 59.9 & $1.59 \pm 0.02$ & $8.85 \pm 0.05$ \\
AUT $=\mathbf{1 7 5 0}$ hours & 19.7 & 30.6 & 47.3 & $3.42 \pm 0.02$ & $18.2 \pm 0.2$ \\
\hline
\end{tabular}

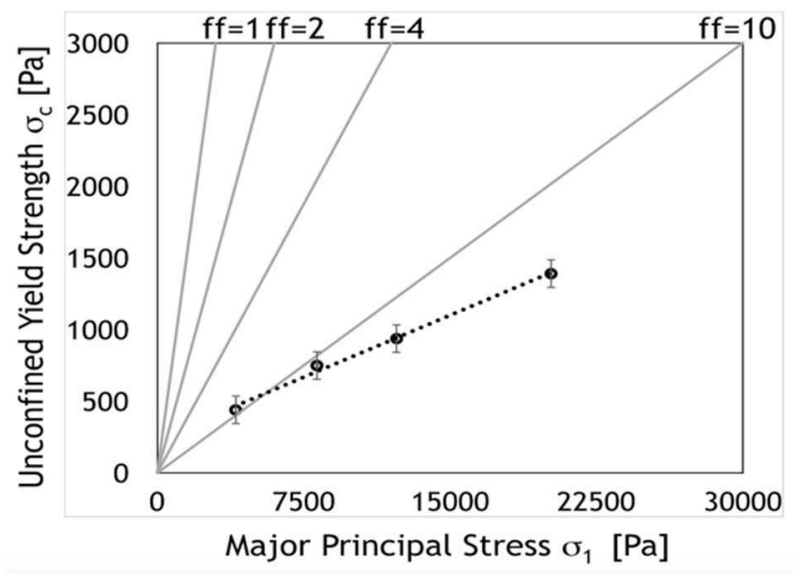

Fig. 5 Flow function of the virgin powder determined according to the ASTM standard D7891-15 [14].

The particle size distribution of the Ti-6Al-4V virgin powder followed a normal gaussian trend. The corresponding characteristic size values are listed in Tab. 2. The results for the Hall and Carney funnel-based flowability tests are detailed in the same Tab. 2. The complete flow function for the feedstock powder is reported in Fig.5. According to the obtained result, the flowability behavior of the powder can be ascribed to the free-flowing type, as proposed by Jenike [18]. In fact, the flow function (ff) values (i.e. the ratio between the major principal, $\sigma_{1}$, and the unconfined yield strength, $\sigma_{c}$ ) are almost always greater than 10 , independently of the used consolidation stress.

\subsection{Characterization of the reused powder}

Due to the limited availability of powder at the end of the recycling test, only selected tests were performed after an $A U T$ of 1750 hours.

The SEM observation, as seen in Fig. 6, shows that occasional morphological changes were caused by recycling, since satellite particles were attached to the powder surface and few aggregates could be encountered in spite of the repeated sieving operations. The local microanalysis performed on these aggregates also revealed an average oxygen content that was higher than that typically encountered on the surface of non-aggregated particles (data not shown). 


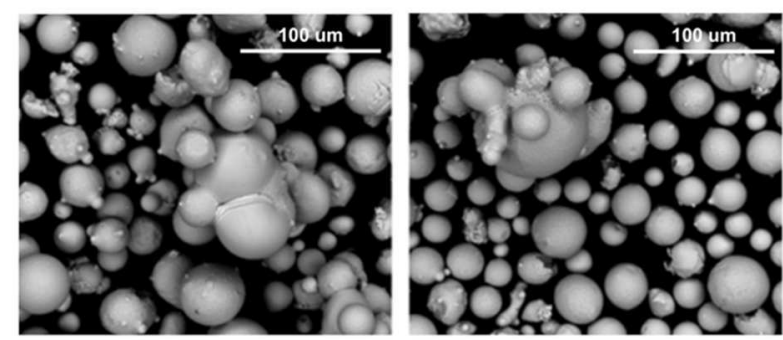

Fig. 6 SEM micrographs of Ti-6Al-4V powder after an AUT of $1750 \mathrm{~h}$.

The $\mathrm{d} 10, \mathrm{~d} 50$ and $\mathrm{d} 90$ values of the particle size distribution of the recycled Ti-6Al-4V powder after an $A U T$ of 1750 hours are detailed in Tab. 2, which also reports the results of flowability tests.

With respect to the virgin powder, the particle size distribution after repeated recycling is slightly finer and the flowability is increased, in spite of the occasional morphological changes described above.

Contrasting results on the effect of recycling on powder size distribution and aggregation can be found in the literature. Ardila et al., for example, analyzed the reuse of nickel alloy 718 powder in laser selective melting and they also observed the progressive formation of melting-induced particle aggregation [19]. Also Seyda et al. [9] observed a progressive but sensible increase in the average particle size of Ti-6Al-4V powder after repeated recycling in powder laser melting and the increased average particle size was attributed to the partial sintering/melting of particles that had occurred close to the melt pool. However, opposite results were reported by other Authors. For example, Slotwinski et al. [20] analyzed the effect of reusing 17-4 stainless steel powder in a commercial direct metal laser sintering apparatus. At the end of each job, Slotwinski et al. sampled some powder from the powder bed directly, which means they considered the particles in close proximity to the completed part, and some powder after sieving. The particle size distribution was the same for the virgin powder and for the reused powder after sieving, whereas the size distribution of the reused powder before sieving was shifted towards finer dimensions. This discrepancy was attributed to the action of the re-coating arm that pushed aside the larger particles, thus removing them from the powder bed.

As a matter of fact, the evolution of the particle size distribution that can be measured after repeated recycling is affected not only by the phenomena directly associated to the building process, such as aggregation, break up or partial sintering, but also by the specific sieving and recycling strategies that are applied in order to reuse the powder [9].

\subsection{Effect of powder recycling on L-PBF parts}

The average density of the L-PBF cubes (calculated on the entire duration of the recycling test) was $4.42 \pm$ $0.01 \mathrm{~g} / \mathrm{cm}^{3}$. Since the density of the cubes showed a very narrow variability throughout the entire recycling test, no statistical analysis was performed to assess the dependence of density on $A U T$. It is worth noting that the average density of the cubes was comparable to the theoretical density of Ti-6Al-4V, which is $4.43-4.45 \mathrm{~g} / \mathrm{cm}^{3}$
[21]. This close correspondence suggests that a full densification was achieved through L-PBF manufacturing.

This information was verified by means of the direct SEM observation of the cross section of the printed cubes. As shown in Fig. 7, the built parts were pore-free and fully dense, in spite of the repeated reuse of the feedstock powder. Moreover the visual inspection through SEM proved the absence of lack of fusion defects. This is an important result, since lack of fusion defects have adverse effects on the mechanical properties of finished parts, but they cannot be revealed through the Archimedes' method [22]. As already seen for the virgin powder, also the cross section of the printed parts exhibited feather-like fine microstructural features, which can be attributed to the coexistence of different titanium phases. The X-EDS microanalysis confirmed the presence of $\mathrm{Ti}, \mathrm{Al}$, and $\mathrm{V}$ (Fig. 3b). No sensible oxidation was observed in the finished parts, which proved that neither the iterated reuse of the feedstock powder nor the powder sieving operations caused any oxidation in the printed parts.
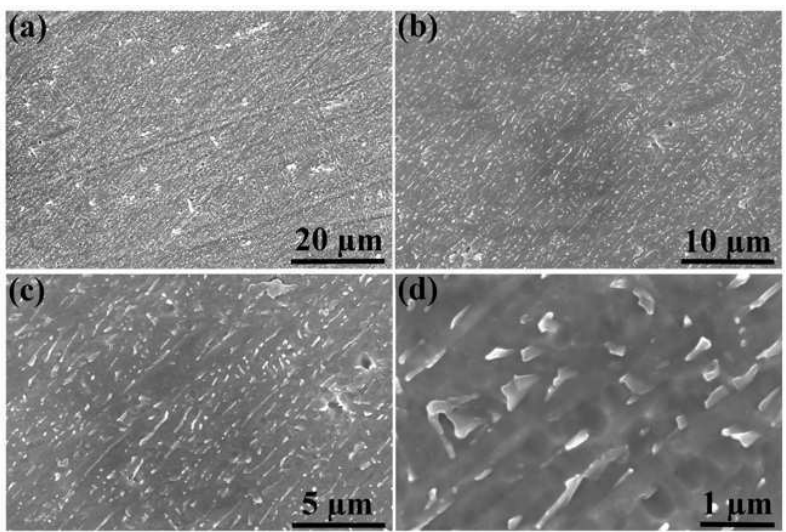

Fig. 7 Microstructure of the printed samples

Coherently with the SEM outcomes, the diffraction pattern of the printed samples fitted by the Rietveld method (Fig. 4b) was compatible with the survival of the two $\alpha$ and $\alpha$ ' crystallographic phases from the virgin powder to the finished part, even after repeated powder reuse and L-PBF processing. The only assessable effect is the difference in the relative quantities of the two phases, being the $\alpha$ phase in the printed sample about $10 \%$ higher than in the powder sample, whereas the distorted phase shows the opposite trend.

Fig. 8 summarizes the average mechanical properties $\left(\mathrm{UTS}=\right.$ ultimate tensile stress; $\mathrm{R}_{\mathrm{p}}=$ yield stress; $\mathrm{A}_{\mathrm{t}}=$ elongation) calculated as the mean value of all tensile specimens throughout the entire recycling test.

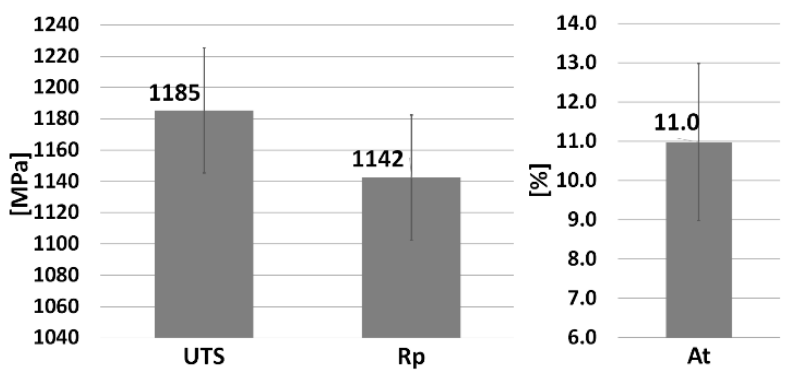

Fig. 8 Results of mechanical tests on tensile samples produced throughout the entire recycling test. 
In order to assess the effect of powder recycling in more detail, the tensile properties were expressed as a function of $A U T$ as defined in Section 2.2. The results are reported in Fig. 9 and the statistical correlations are summarized in Tab. 3. AUT represents here an easy parameter to express the duration of powder usage in quantitative terms. This parameter is very effective to account for the real recycling strategy in industry, since it can be used to describe flexible build jobs and repeated refilling operations.

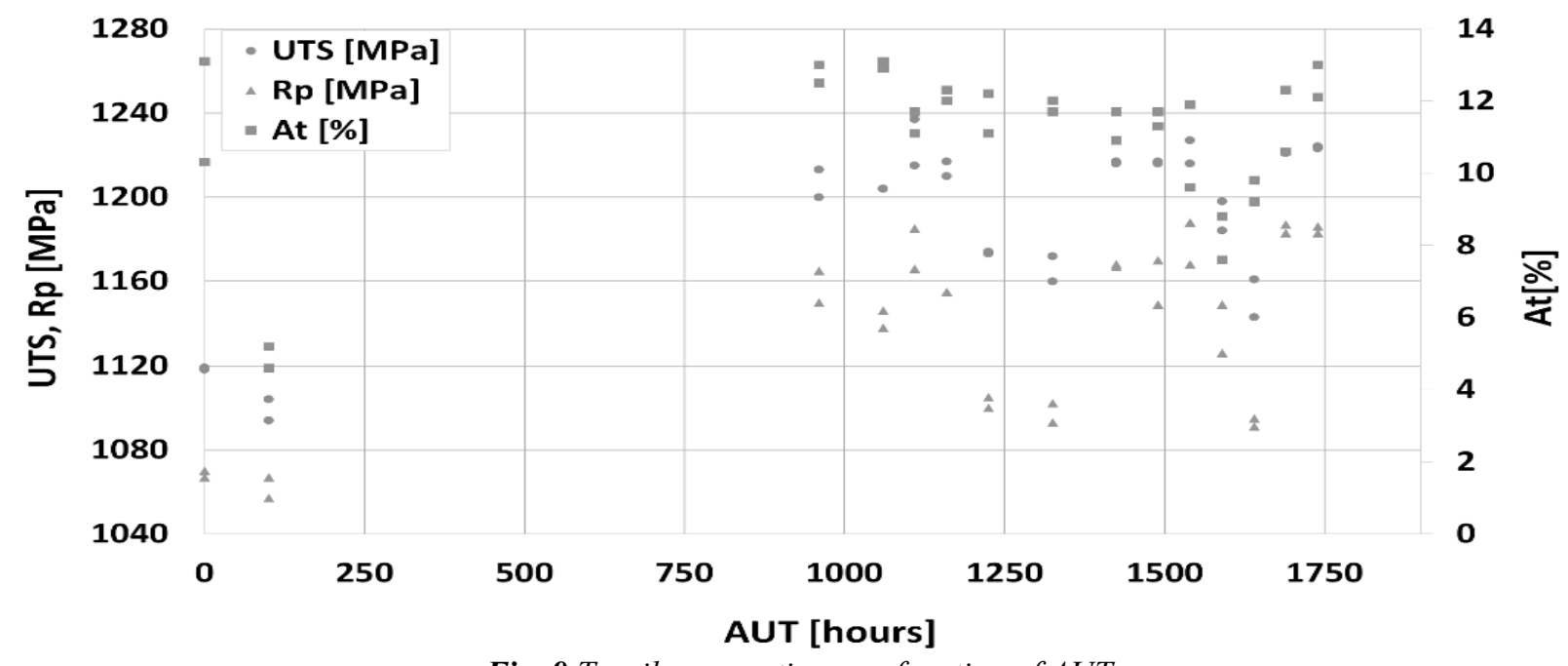

Fig. 9 Tensile properties as a function of AUT.

Tab. 3 Statistical correlations between tensile properties and AUT. Correlation coefficients having p-values $<0.0001$ are written in bold.

\begin{tabular}{lccc}
\hline & $\mathbf{R}_{\mathbf{p}}$ & $\mathbf{U T S}$ & $\mathbf{A}_{\mathbf{t}}$ \\
\hline \multirow{2}{*}{ All samples } & $\mathbf{0 . 6 6}$ & $\mathbf{0 . 7 2}$ & 0.29 \\
& $(\mathbf{p}<\mathbf{0 . 0 0 0 1})$ & $(\mathbf{p}<\mathbf{0 . 0 0 0 1 )}$ & $(\mathrm{p}=0.120)$ \\
Samples position 3 & $\mathbf{0 . 9 0}$ & $\mathbf{0 . 9 4}$ & 0.26 \\
& $(\mathbf{p}<\mathbf{0 . 0 0 2})$ & $(\mathbf{p}=\mathbf{0 . 0 0 0 7 )}$ & $(\mathrm{p}=0.241)$ \\
\hline
\end{tabular}

As shown in Tab. 3, a strong correlation with $A U T$ was revealed for $\mathrm{R}_{\mathrm{p}}$ and UTS. Even if the trend may seem counterintuitive, $\mathrm{R}_{\mathrm{p}}$ and UTS didn't decrease as $A U T$ increased. In other words, in spite of the data scattering, it is worth noting that neither $\mathrm{R}_{\mathrm{p}}$ nor UTS decreased as a result of the long reuse of the feedstock powder.

The statistical analysis was repeated by taking into account the tensile samples produced in a specific position of the build platform, so as to highlight any possible side effects associated to relative position. In fact, the outcome of L-PBF processing may be affected by position-specific conditions such as different gas flow speed or non-homogenous powder spreading. In more detail, Tab. 3 includes the statistical correlations between $A U T$ and the tensile properties of the specimens produced in the position " 3 " of the build platform as defined in Fig. 1. Although the statistical analysis was addressed to the samples built in the position " 3 " only, a strong correlation with $A U T$ was revealed for $R_{p}$ and UTS. In fact, neither $R_{p}$ nor UTS decreased as a result of powder reuse. Even if additional data would be beneficial in order to confirm this trend, it is interesting to note that this result seems to contradict the intuitive expectation that mechanical properties should be negatively affected by powder reuse. On the other hand, this outcome is coherent with the results published by Ardila et al. [19] who didn't observe any change in Charpy impact strength after reusing the same nickel alloy 718 powder through 14 jobs (without refilling) in selective laser melting. Tang et al. [10] investigated the effect of Ti-6Al-4V powder reuse in selective electron beam melting and they observed an increase in the oxygen content of the recycled powder. However, the repeated powder reuse didn't affect negatively the mechanical properties of the tensile samples. Similar conclusions were achieved also by Hann [5], who demonstrated that, after 10 jobs completed by reusing the same nickel alloy 718 powder, there were no consequences on the chemistry and size distribution of the powder, nor on the mechanical properties of the finished parts processed by LPBF.

\section{Conclusions}

The average usage time (AUT) is an innovative parameter that takes into account two key factors associated to powder recycling, namely (i) the repeated use of powder through different jobs that may differ in duration and geometry and (ii) the progressive addition of virgin powder to restore the amount of feedstock that is required to complete the jobs. In this way, AUT can be a practical parameter for industrial applications, since it sums up various phenomena that are expected to take place during processing, such as fast heating and cooling that are specially relevant for those particles that are close to the part in the build chamber, as well as oxidation, partial or complete melting, or sintering. Though introduced here for laser-based powder bed fusion, in principle $A U T$ can be easily extended to other additive manufacturing methods, thus providing a straightforward tool to analyze the effect of feedstock recycling. Interestingly, the tensile tests proved that yield stress and ultimate tensile stress didn't 
decrease as a consequence of powder recycling, although the particle size distribution was slightly finer after repeated reuse and also the morphology of the powder was occasionally modified due to the presence of few satellite particles and aggregates.

\section{References}

[1] KRUTH, J.P., WANG, X., LAOUI, T., FROYEN, L. (2003). Lasers and materials in selective laser sintering. In: Assembly Autom, Vol. 23, pp. 357 371.

[2] TONG, J., BOWEN, C.R., PERSSON, J., PLUMMER, A. (2016). Mechanical properties of titanium-based Ti-6Al-4V alloys manufactured by powder bed additive manufacture. In: Mater Sci Tech, Vol. 33, pp. 11.

[3] FOUSOVÁ, M., VOJTĚCH, D., KUBÁSEK, J. (2016). Titanium alloy Ti-6Al-4V prepared by selective laser melting (SLM). In: Manufacturing Technology, Vol. 16, pp. $691-697$.

[4] FOUSOVÁ, M., VOJTECH, D., (2017). Influence of process conditions on additive manufacture of Ti6Al4V alloy by SLM technology. In: Manufacturing Technology, Vol. 17, pp. 696- 701.

[5] HANN, B. (2016). Powder reuse and its effects on Laser Based Powder Fusion Additive Manufactured Alloy 718. In: SAE Int J Aerosp, Vol. 9, pp. $209-213$.

[6] SEIFI, M., SALEM, A., BEUTH, J., HARRYSSON, O., LEWANDOWSKI, J.J. (2016). Overview of materials qualification needs for metal additive manufacturing. In: JOM, Vol. 68 , pp. $747-764$

[7] ASTM F2924-14, Standard Specification for Additive Manufacturing Titanium-6 Aluminum-4 Vanadium with Powder Bed Fusion. (2014). ASTM International, West Conshohocken, PA.

[8] BRICÍN, D., KŘíŽ, A. (2018). Assessment of Usability of WC-Co Powder Mixtures for SLM. In: Manufacturing Technology, Vol. 18, 719 726.

[9] SEYDA, V., KAUFMANN, N., EMMELMANN, C. (2012). Investigation of aging processes of Ti$6 \mathrm{Al}-4 \mathrm{~V}$ powder material in laser melting. In: Physics procedia of the 7th international conference $\&$ exhibition on photonic technologies LANE, vol. 39, Fürth, Germany, p. 425 - 431.

[10] TANG, H.P., QIAN, M., LIU, N., ZHANG, X.Z., YANG, G.Y., WANG, J. (2015). Effect of powder reuse times on additive manufacturing of Ti-6Al$4 \mathrm{~V}$ by selective electron beam melting. In: JOM, Vol. 67, pp. $555-563$.

[11] ASTM B822-17, Standard Test Method for Particle Size Distribution of Metal Powders and Related Compounds by Light Scattering. (2017). ASTM International, West Conshohocken, PA.
[12] ASTM B213-17, Standard Test Methods for Flow Rate of Metal Powders Using the Hall Flowmeter Funnel. (2017). ASTM International, West Conshohocken, PA.

[13] ASTM B964-16, Standard Test Methods for Flow Rate of Metal Powders Using the Carney Funnel. (2016). ASTM International, West Conshohocken, PA.

[14] ASTM D7891-15, Standard Test Method for Shear Testing of Powders Using the Freeman Technology FT4 Powder Rheometer Shear Cell. (2015). ASTM International, West Conshohocken, PA.

[15] EOS Titanium Ti64, Material Data Sheet, at: https://cdn.eos.info/a4eeb73865d54434/5926811 b3739/Ti-Ti64 9011-0014 90110039_M290_Material_data_sheet_11-17_en.pdf; last accessed: May 31, $201 \overline{8}$.

[16] ISO. Metallic Materials-Tensile Testing-Part 1: Method of Test at Room Temperature. (2016). UNI EN ISO 6892-1; International Organization for Standardization: Geneva, Switzerland.

[17] NOURI, A., SOLA, A. (2018). Metal particle shape: A practical perspective. In: Met Powder Rep, Vol. 73, pp. $276-282$.

[18] JENIKE, A.W. Storage and flow of solids. (1964). In: Bulletin of the University of Utah 123, Vol. 53, pp. 207.

[19] ARDILA, L.C., GARCIANDIA, F., GONZÁLEZ DÍAZ, J.B., ÁLVAREZ, P., ECHEVERRIA, A., PETITE, M.M., DEFFLEY, R., OCHOA, J. Effect of IN718 recycled powder reuse on properties of parts manufactured by means of Selective Laser Melting. (2014). In: Physics procedia of the 8th international conference on photonic technologies LANE, vol. 56, Fürth, Germany, p. 99 - 107.

[20] SLOTWINSKI, J.A., GARBOCZI, E.J., STUTZMAN, P.E., FERRARIS, C.F., WATSON, S.S., PELTZ, M.A. Characterization of metal powders used for additive manufacturing. (2014). In: J Res Natl Inst Stand Technol, Vol. 119, pp. $460-493$.

[21] ASM Aerospace Specification Metals Inc., available on-line at http://asm.matweb.com/search/SpecificMaterial.asp?bassnum $=$ mtp641; last accessed: June 1, 2018.

[22] GONG, H., RAFI, K., GU, H., JANAKI RAM, G.D., STARR, T., STUCKER, B. Influence of defects on mechanical properties of $\mathrm{Ti}-6 \mathrm{Al}-4 \mathrm{~V}$ components produced by selective laser melting and electron beam melting. (2015). In: Mater Des, Vol. 86, pp. $545-555$. 